\title{
Autopercepción de capacidades de autocuidado para la prevención de enfermedades no transmisibles en universitarios
}

\author{
Fabiola Villegas Pérez \\ fabiperez1297@gmail.com
}

\begin{abstract}
Alma Rosa García Hernández almag7601@gmail.com
\end{abstract}

Dra. Ivett Rodríguez Muñoz iverodriguez@uv.mx

Dr. Javier Salazar Mendoza* jasalazar@uv.mx https://orcid.org/0000-0001-9172-8731

MCE. Minerva Peña López minpena@uv.mx

\section{MCE. María de los Ángeles Onofre Santiago aonofre@uv.mx https://orcid.org/0000-0002-8951-4379}

MCE. María Esperanza Conzatti Hernández econzatti@uv.mx https://orcid.org/0000-0002-9612-2613

Universidad Veracruzana, Facultad de Enfermería Orizaba

\section{RESUMEN}

Introducción: actualmente las enfermedades no transmisibles son una de las principales problemáticas de salud de América Latina debido a su alta tasa de mortalidad, en México, la morbilidad de éstas va en aumento en la población más joven, por esta razón la autopercepción de autocuidado juega un papel importante en la prevención puesto que, los universitarios tienden a cambiar sus hábitos diarios al ingresar a la universidad. Objetivo: Determinar el nivel de autopercepción de la capacidad de autocuidado para la prevención de las enfermedades no transmisibles en estudiantes de la facultad de enfermería de la Universidad Veracruzana. Estrategias metodológicas: investigación cuantitativa, descriptiva, prospectiva, transversal y no experimental en una muestra de 195 estudiantes, con muestreo no probabilístico por cuota, utilizando el cuestionario conductas de autocuidado, el análisis de datos se realizó a través del paquete estadístico 
SPSS. Resultados: la autopercepción de autocuidado en el $95.9 \%$ fue muy buena y buena, el $85.6 \%$ tuvo un nivel de conocimiento bueno de exámenes de salud preventivos. Conclusión: el 56.9\% posee un peso normal, respecto a la actividad física el $43.6 \%$ no la realiza por falta de tiempo, un $93.8 \%$ no consume drogas, en cuanto a exámenes preventivos el $62.6 \%$ los lleva a cabo.

Palabras clave: autopercepción; autocuidado; enfermedades no transmisibles. 


\title{
Self-perception of self-care capabilities for the prevention of noncommunicable diseases in university students
}

\begin{abstract}
Introduction: currently, non-communicable diseases are one of the main health problems in Latin America due to their high mortality rate; in Mexico, morbidity of these diseases is increasing in the younger population. For this reason, self-care self-perception plays an important role in prevention since university students tend to change their daily habits when they enter university. Objective: To determine the level of self-perception of selfcare capacity for the prevention of non-communicable diseases in students of the nursing faculty of the Universidad Veracruzana. Methodological strategies: quantitative, descriptive, prospective, cross-sectional, and non-experimental research in a sample of 195 students, with non-probabilistic sampling by quota, using the self-care behaviors questionnaire; data analysis was performed through the SPSS statistical package. Results: the self-perception of self-care in $95.9 \%$ was very good and good, $85.6 \%$ had a good level of knowledge of preventive health examinations. Conclusion: $56.9 \%$ had a normal weight, $43.6 \%$ did not engage in physical activity due to lack of time, $93.8 \%$ did not use drugs, and $62.6 \%$ underwent preventive examinations.
\end{abstract}

Key words: self-perception, self-care, non-communicable diseases.

Artículo recibido: 05 octubre. 2021 Aceptado para publicación: 02 noviembre 2021

Correspondencia: jasalazar@uv.mx Conflictos de Interés: Ninguna que declarar 


\section{INTRODUCCIÓN}

Actualmente el rol que tiene la profesión de enfermería en las disciplinas del área de la salud y sociedad está definido por su trayectoria y quehacer en el transcurso del tiempo, influenciado por una serie de hechos y circunstancias que han definido su actuar y su función social.

Enfermería, es una disciplina que exige conocimientos y compromiso ante la autopercepción la cual se define como la concepción individual y subjetiva entre factores biológicos, sociales y psicológicos, que proporciona una evaluación única del estado general de salud de una persona (Bustos, Fernández \& Astudillo, 2017) si bien es necesario que toda persona que pertenece a la profesión cuente con un estado de salud de calidad y sobre todo autocuidado ya que mantiene una relación con la autopercepción (Naranjo, Concepción \& Rodríguez, 2020) función humana reguladora que debe aplicar cada individuo de forma deliberada con el fin de mantener su vida y su estado de salud, desarrollo y bienestar.

Según la Organización Mundial de la Ssalud (2018), las enfermedades no transmisibles (ENT) tienden a ser de larga duración y resultan de la combinación de factores genéticos, fisiológicos, ambientales y conductuales. Entre las principales se encuentran las cardiovasculares, respiratorias crónicas, el cáncer y la diabetes, que en conjunto son responsables de más del $80 \%$ de todas las muertes prematuras y ocasionan anualmente cerca de 41 millones de defunciones, cifra que representa el $71 \%$ de la mortalidad global. Por lo anterior, es de gran interés comprobar si los universitarios tienen una buena autopercepción de autocuidado antes las ENT, dado que la población joven es la fuerza del país. En México en 2019, había un registro de 37, 504, 392 jóvenes de 12 a 29 años, representando el $31.4 \%$ de la población total y según el boletín del sistema epidemiológico la incidencia de enfermedades no transmisibles fue de 684,929 casos y desafortunadamente va en aumento esta proporción entre la población más joven (Secretaría de salud, 2019).

Por lo anterior se plantea como objetivo: determinar el nivel de autopercepción de la capacidad de autocuidado para prevención de enfermedades no transmisibles en estudiantes de la facultad de enfermería de la Universidad Veracruzana. 


\section{ESTRATEGIAS METODOLÓGICAS}

Estudio cuantitativo, descriptivo y transversal (Hernández, Alvarado \& Pineda, 2013; Hernández, Fernández \& Baptista, 2014), en una población de 412 alumnos se obtuvo una muestra de 195 estudiantes de tercer, quinto y séptimo semestre, de la Facultad de Enfermería Orizaba de la Universidad Veracruzana, tomando como referencia la fórmula de Fisher y Navarro (Villareal, 2015) con un 95\% de coeficiente de nivel de confianza. La obtención de la muestra fue a través del método de muestreo no probabilístico por cuota (Hernández et al., 2013) y en apego a los criterios de selección se incluyeron alumnos con matrícula 1900, 1800 y 1700, que aceptaran participar en el cuestionario, con consentimiento informado el cual fue enviado vía electrónica y devuelto de la misma manera. Se excluyeron alumnos de nuevo ingreso, de servicio social y quien padecieran una patología crónica, se eliminaron las pruebas que no fueron llenados correctamente o que estuvieron incompletos (Tamayo, 2014). El instrumento de medición fue el de conductas de autocuidado (Martínez \& Sáez, 2007) se adaptó en su versión original a la electrónica en la plataforma de Google formularios, se integra por cinco dimensiones: antecedentes personales, salud mental, hábitos de vida saludable, salud sexual, autoexamen de mamas y testicular. La escala de medición tiene un patrón de respuesta de 0 a 2 donde, $0=$ casi siempre, $1=$ a veces y $2=$ nunca.

Esta investigación fue aprobada por el Comité de bioética de la Facultad de Enfermería de Orizaba. Las personas que decidieron participar aceptaron un consentimiento informado vía electrónica, para garantizar la confidencialidad la información que se obtuvo de forma anónima, el participante se registró con un código por lo que no se puede, asociar o conectar con la persona a quien se refiere. Así como lo sugiere recomendaciones "Principios éticos aplicados a la epidemiología. Pautas Internacionales para la Evaluación Ética de los Estudios Epidemiológicos CIOMS" (Gobierno de México, 2019).

No se expuso a la persona a riesgos ni daños innecesarios de acuerdo con la normativa nacional vigente y los beneficios esperados son mayores que los riesgos predecibles según la Norma Oficial Mexicana NOM-012-SSA3-2012, que establece los criterios para la ejecución de proyectos de investigación para la salud en seres humanos (DOF, 2013).

Para elaborar el análisis de la información, se utilizó el programa estadístico Statiscal Package for the Social Sciences (SPSS) versión 20 para Windows (SPSS Inc, 2006), creando una base de datos donde se capturaron los instrumentos, previa validación y 
revisión del llenado completo y correcto de ellos. El plan de análisis estuvo integrado por estadística descriptiva (Celis \& Labrada, 2014) mediante el uso de frecuencia, porcentajes.

\section{RESULTADOS Y DISCUSIÓN}

La Tabla 1 contiene los datos sociodemográficos donde hay un total de población de 195 alumnos, de estos 158 son mujeres y 37 varones, dato que confirma que la profesión de enfermería sigue demandada mayormente por la población femenina.

En cuanto a la edad que predomina se encuentra entre los 20 a 23 años con un $66.7 \%$, edad en que el individuo obtiene su formación profesional en México y en su mayoría, no tiene responsabilidades de una familia, ya que al analizar la información se determinó que el $92.8 \%$ son personas solteras, mientras que el $73.3 \%$ profesan la religión católica, es decir, mantiene una fe activa que les permite recurrir a ello. Respecto al lugar de procedencia un $76.4 \%$ del total de universitarios pertenece a la zona urbana.

\section{Tabla 1}

Datos sociodemográficos de los estudiantes

\begin{tabular}{|c|c|c|c|c|c|c|}
\hline \multirow{3}{*}{ Edad en años } & \multicolumn{4}{|c|}{ Género } & \multirow{2}{*}{\multicolumn{2}{|c|}{ Total }} \\
\hline & \multicolumn{2}{|c|}{$\begin{array}{c}\text { Mujer } \\
(\mathrm{n}=158)\end{array}$} & \multicolumn{2}{|c|}{$\begin{array}{c}\text { Hombre } \\
(\mathrm{n}=37)\end{array}$} & & \\
\hline & $f$ & $\%$ & $f$ & $\%$ & $f$ & $\%$ \\
\hline $18-19$ & 27 & 13.8 & 6 & 3.1 & 33 & 16.9 \\
\hline $20-21$ & 66 & 33.8 & 5 & 2.6 & 71 & 36.4 \\
\hline $22-23$ & 43 & 22.1 & 16 & 8.2 & 59 & 30.3 \\
\hline $24-25$ & 11 & 5.6 & 6 & 3.1 & 17 & 8.7 \\
\hline $26-27$ & 6 & 3.1 & 2 & 1.0 & 8 & 4.1 \\
\hline $28-29$ & 3 & 1.5 & 0 & 0 & 3 & 1.5 \\
\hline $30-31$ & 2 & 1.0 & 2 & 1.0 & 4 & 2.1 \\
\hline \multicolumn{7}{|l|}{ Estado civil } \\
\hline Soltero & 146 & 74.9 & 35 & 17.9 & 181 & 92.8 \\
\hline Casado & 6 & 3.1 & 2 & 1.0 & 8 & 4.1 \\
\hline Unión libre & 6 & 3.1 & 0 & 0 & 6 & 3.1 \\
\hline \multicolumn{7}{|l|}{ Religión } \\
\hline Católica & 114 & 58.5 & 29 & 14.9 & 143 & 73.3 \\
\hline Cristiana & 27 & 13.8 & 21 & 0.5 & 28 & 14.4 \\
\hline Ninguna & 10 & 5.1 & 4 & 2.1 & 14 & 7.2 \\
\hline Otra & 6 & 3.5 & 3 & 1.5 & 9 & 5.0 \\
\hline \multicolumn{7}{|c|}{ Lugar de procedencia } \\
\hline Rural & 41 & 21.0 & 5 & 2.6 & 46 & 23.6 \\
\hline Urbano & 117 & 60.0 & 32 & 16.4 & 149 & 76.4 \\
\hline Total & 158 & $81.0 \%$ & 37 & $19.0 \%$ & 195 & $100.0 \%$ \\
\hline
\end{tabular}




\section{Tabla 2}

Nivel de autopercepción de autocuidado por género

\begin{tabular}{|c|c|c|c|c|c|c|}
\hline \multirow{3}{*}{ Nivel } & \multicolumn{4}{|c|}{ Género } & \multirow{2}{*}{\multicolumn{2}{|c|}{ Total }} \\
\hline & \multicolumn{2}{|c|}{$\begin{array}{c}\text { Mujer } \\
(n=158)\end{array}$} & \multicolumn{2}{|c|}{$\begin{array}{c}\text { Hombre } \\
(n=37)\end{array}$} & & \\
\hline & $f$ & $\%$ & $f$ & $\%$ & $f$ & $\%$ \\
\hline Muy bueno & 78 & 40.0 & 14 & 7.2 & 92 & 47.2 \\
\hline Bueno & 73 & 37.4 & 22 & 11.3 & 95 & 48.7 \\
\hline Regular & 7 & 3.6 & 1 & 0.5 & 8 & 4.1 \\
\hline Total & 158 & $81.0 \%$ & 37 & $19.0 \%$ & 195 & $100.0 \%$ \\
\hline
\end{tabular}

Fuente: Encuesta sobre conductas de autocuidado.

La Tabla 2 determina el nivel de autopercepción de autocuidado por género de los estudiantes que participaron en este estudio, se obtuvo que la clasificación muy buena en el $47.2 \%$, de éstas y tomando al género como el $100 \%$, en el $49.3 \%$ de la mujeres lo experimentó, mientras que en el $37.8 \%$ fueron hombres, por otro lado, el $48.7 \%$ de autopercepción general fue bueno y $4.1 \%$ regular.

\section{Tabla 3}

Conductas de autocuidado del sueño y descanso

\begin{tabular}{|c|c|c|c|c|c|c|}
\hline \multirow{3}{*}{$\begin{array}{l}\text { Duerme bien y se siente } \\
\text { descansado }\end{array}$} & \multicolumn{4}{|c|}{ Género } & \multirow{2}{*}{\multicolumn{2}{|c|}{ Total }} \\
\hline & \multicolumn{2}{|c|}{$\begin{array}{c}\text { Mujer } \\
(n=158)\end{array}$} & \multicolumn{2}{|c|}{$\begin{array}{c}\text { Hombre } \\
(n=37)\end{array}$} & & \\
\hline & $f$ & $\%$ & $f$ & $\%$ & $f$ & $\%$ \\
\hline Casi siempre & 24 & 12.3 & 8 & 4.1 & 32 & 16.4 \\
\hline A veces & 118 & 60.5 & 25 & 12.8 & 143 & 73.3 \\
\hline Nunca & 16 & 8.2 & 4 & 2.1 & 20 & 10.3 \\
\hline \multicolumn{7}{|c|}{ Promedio de horas que duerme diariamente } \\
\hline Entre 4 y 6 horas & 82 & 42.1 & 18 & 9.2 & 100 & 51.3 \\
\hline Entre 7 y 8 horas & 71 & 36.4 & 17 & 8.7 & 88 & 45.1 \\
\hline Más de 8 horas & 5 & 2.6 & 2 & 1.0 & 7 & 3.6 \\
\hline Total & 158 & $81.0 \%$ & 37 & $19.0 \%$ & 195 & $100.0 \%$ \\
\hline
\end{tabular}

Fuente: Encuesta sobre conductas de autocuidado.

La Tabla 3 analiza las conductas de autocuidado del sueño y descanso, el $73.3 \%$ a veces duermen bien y se siente descansado, sin embargo, el $51.3 \%$ solo duerme entre 4 y 6 horas diariamente y un $45.1 \%$ entre 7 y 8 horas. 
Tabla 4

Manejo del estrés

\begin{tabular}{lcccccc}
\hline \multirow{2}{*}{$\begin{array}{l}\text { Sentirse } \\
\text { estresado }\end{array}$} & \multicolumn{3}{c}{$\begin{array}{c}\text { Mujer } \\
(\mathbf{n = 1 5 8 )}\end{array}$} & \multicolumn{2}{c}{$\begin{array}{c}\text { Hombre } \\
(\boldsymbol{n}=\mathbf{3 7})\end{array}$} & \multicolumn{2}{c}{ Total } \\
\cline { 2 - 6 } & $\boldsymbol{f}$ & $\mathbf{\%}$ & $\boldsymbol{f}$ & $\mathbf{\%}$ & $\boldsymbol{f}$ & $\mathbf{\%}$ \\
\hline Casi siempre & 64 & 32.8 & 4 & 2.1 & 68 & 34.9 \\
A veces & 93 & 47.7 & 31 & 15.9 & 124 & 63.6 \\
Nunca & 1 & 0.5 & 2 & 1.0 & 3 & 1.5 \\
Manejar el estrés & $\mathbf{0}$ & tensión en su vida & & & & \\
Casi siempre & 52 & 26.7 & 19 & 9.7 & 71 & 36.4 \\
A veces & 102 & 52.3 & 17 & 8.7 & 119 & 61.0 \\
Nunca & 4 & 2.1 & 1 & 0.5 & 5 & 2.6 \\
\hline Total & $\mathbf{1 5 8}$ & $\mathbf{8 1 . 0 \%}$ & $\mathbf{3 7}$ & $\mathbf{1 9 . 0 \%}$ & $\mathbf{1 9 5}$ & $\mathbf{1 0 0 . 0 \%}$ \\
\hline
\end{tabular}

Fuente: Encuesta sobre conductas de autocuidado.

En la Tabla 4 se estudió la frecuencia de sentirse estresado y el manejo de la tensión en la vida, obteniendo como resultado que el $63.3 \%$ del total de la población se sienten a veces estresados, de los cuales manejan el estrés o tensión un $61 \%$, destacando que el total de universitarios que casi siempre esta estresado es el $34.9 \%$ y el estudio demostró que el $36.4 \%$ casi siempre sabe manejar el estrés o tensión en su vida.

\section{Tabla 5}

Estado nutricional

\begin{tabular}{|c|c|c|c|c|c|c|}
\hline \multirow{3}{*}{ Clasificación del IMC } & \multicolumn{4}{|c|}{ Género } & \multirow{2}{*}{\multicolumn{2}{|c|}{ Total }} \\
\hline & \multicolumn{2}{|c|}{$\begin{array}{c}\text { Mujer } \\
(n=158)\end{array}$} & \multicolumn{2}{|c|}{$\begin{array}{c}\text { Hombre } \\
(n=37)\end{array}$} & & \\
\hline & $f$ & $\%$ & $f$ & $\%$ & $f$ & $\%$ \\
\hline Bajo peso & 5 & 2.6 & 2 & 1.0 & 7 & 3.6 \\
\hline Normal & 89 & 45.6 & 22 & 11.3 & 111 & 56.9 \\
\hline Sobrepeso & 48 & 24.6 & 9 & 4.6 & 57 & 29.2 \\
\hline Obesidad grado I & 16 & 8.2 & 4 & 2.1 & 20 & 10.2 \\
\hline Total & 158 & $81.0 \%$ & 37 & $19.0 \%$ & 195 & $100.0 \%$ \\
\hline
\end{tabular}

Fuente: Encuesta sobre conductas de autocuidado.

La Tabla 5 integra el estado nutricional, según el índice de masa corporal de los universitarios el $56.9 \%$ se encuentra en su peso normal, en sobrepeso se localiza al $29.2 \%$ del total de la población encuestada. Cabe mencionar que el $10.2 \%$ de universitarios se encuentra en obesidad y el $3.6 \%$ tiene bajo peso. 
Tabla 6

Consumo de drogas y alcohol

\begin{tabular}{|c|c|c|c|c|c|c|}
\hline \multirow{3}{*}{$\begin{array}{c}\text { Número de vasos que } \\
\text { bebe por semana }(200 \mathrm{ml})\end{array}$} & \multicolumn{4}{|c|}{ Género } & \multirow{2}{*}{\multicolumn{2}{|c|}{ Total }} \\
\hline & \multicolumn{2}{|c|}{$\begin{array}{c}\text { Mujer } \\
(n=158)\end{array}$} & \multicolumn{2}{|c|}{$\begin{array}{c}\text { Hombre } \\
(n=37)\end{array}$} & & \\
\hline & $f$ & $\%$ & $f$ & $\%$ & $f$ & $\%$ \\
\hline Ninguno & 157 & 80.5 & 36 & 18.5 & 133 & 68.2 \\
\hline De 1 a 12 Vasos & 1 & 0.5 & 1 & 0.5 & 37 & 19.0 \\
\hline Más de 12 vasos & 0 & 0 & 0 & 0 & 25 & 12.8 \\
\hline \multicolumn{7}{|c|}{ Consume Marihuana, Cocaína y/o Pasta Base } \\
\hline Nunca & 150 & 76.9 & 33 & 16.9 & 183 & 93.8 \\
\hline Ocasionalmente & 5 & 2.6 & 2 & 1.0 & 7 & 3.6 \\
\hline A veces & 3 & 1.5 & 2 & 1.0 & 5 & 2.6 \\
\hline \multicolumn{7}{|c|}{ Consumo de Café, té y bebidas colas al día (200 ml) } \\
\hline Nunca & 132 & 67.7 & 30 & 15.4 & 162 & 83.1 \\
\hline Ocasionalmente & 23 & 11.8 & 7 & 3.6 & 30 & 15.4 \\
\hline A veces & 3 & 1.5 & 0 & 0 & 3 & 1.5 \\
\hline Total & 158 & $81.0 \%$ & 37 & $19.0 \%$ & 195 & $100.0 \%$ \\
\hline
\end{tabular}

Fuente: Encuesta sobre conductas de autocuidado.

La Tabla 6, analiza la práctica de toxicomanías, se estudiaron 3 parámetros donde se obtuvo que el $68.2 \%$ de los universitarios no consumen alcohol mientras que el $31.8 \%$ si. El 93.8\% marihuana, cocaína y/o pasta base, los resultados reflejan que el 83.1\% del total nunca ingiere cafeína y $15.4 \%$ si, ocasionalmente.

\section{Tabla 7}

Nivel de conocimiento de autocuidado sobre autoexámenes de salud preventivos

\begin{tabular}{|c|c|c|c|c|c|c|}
\hline \multirow{3}{*}{ Conocimiento } & \multicolumn{4}{|c|}{ Género } & \multirow{2}{*}{\multicolumn{2}{|c|}{ Total }} \\
\hline & \multicolumn{2}{|c|}{$\begin{array}{c}\text { Mujer } \\
(n=158)\end{array}$} & \multicolumn{2}{|c|}{$\begin{array}{c}\text { Hombre } \\
(n=37)\end{array}$} & & \\
\hline & $f$ & $\%$ & $f$ & $\%$ & $f$ & $\%$ \\
\hline Malo & 1 & 0.5 & 0 & 0 & 1 & 0.5 \\
\hline Regular & 23 & 11.8 & 4 & 2.1 & 27 & 13.8 \\
\hline Bueno & 134 & 68.7 & 33 & 16.9 & 167 & 85.6 \\
\hline Total & 158 & $81.0 \%$ & 37 & $19.0 \%$ & 195 & $100.0 \%$ \\
\hline
\end{tabular}

Fuente: Encuesta sobre conductas de autocuidado.

La Tabla 7 evalúa el nivel de conocimiento de autocuidado de los estudiantes en relación con autoexámenes de salud preventivos, los resultados muestran que un $85.6 \%$ del total de encuestados su nivel es bueno y un $13.8 \%$ regular. 
La autopercepción de capacidades de autocuidado ha tenido gran impacto en la vida de las personas, pues es una concepción individual de cada ser humano que tiene acerca de su salud (Naranjo et al., 2020), y aunque cada vez es más grande la tasa de mortalidad por enfermedades no transmisibles que pueden prevenirse, las personas no ejercen un adecuado autocuidado para fortalecer o restablecer la salud y prevenir la enfermedad (OMS, 2018).

Al comparar estos criterios con otros estudios se encuentra que en el trabajo de investigación "Nivel de autocuidado en estudiantes universitarios de cinco licenciaturas del área de la salud" de los autores Simon, López, Sandoval y Magaña, realizado en el año 2016, se relacionaron los factores sociodemográficos edad y religión, ya que en ambos estudios prevalece la edad de 18 a 21 años y el estado civil soltero(a).

También en otro estudio titulado "Autopercepción de capacidades de autocuidado para prevención de enfermedades crónicas no transmisibles en estudiantes universitarios" del autor Rivas et al. realizado en el año 2019, tuvo concordancia en cuanto al género femenino como sobresaliente. Por su parte, el presente estudio solicitó a los entrevistados información acerca de sus capacidades de autocuidado ante enfermedades no transmisibles, brindando como resultado una buena autopercepción que concuerda con los dos estudios anteriormente mencionados, en los que en ambos la autopercepción reflejada fue buena.

En los resultados de esta investigación se dio a conocer que 59\% de los universitarios a veces se sienten satisfechos con su condición física, dato que se contradice con el estudio de Hernández et al. (2018), donde 51.9\% de los encuestados contestaron sentirse bien consigo mismos.

En lo que respecta a la actividad física, este estudio demostró que el $43.6 \%$ no la realiza, ya que un $88.2 \%$ mencionó que es por no tener tiempo, y se ha observado una clara relación con el estudio realizado por Sancho en el 2016 en el cual se expuso que el rango de tiempo de actividad física y cantidad de días por semana van dirigidas de acuerdo con las condiciones de salud de cada persona, así mismo, tiene concordancia con el porcentaje de un $70 \%$ de la población que sí conoce sobre el autocuidado de salud al igual que el resultado reflejado en esta investigación.

El estudio de Nyombi et al. (2016) evidenció la prevalencia de IMC en cuanto a sobrepeso y obesidad que combinados fue del 9.4\%, comparado con esta investigación, se observa 
que el $39.40 \%$ tiene sobrepeso u obesidad, cifra que representa una cantidad mayor de personas con exceso de su peso ideal.

De acuerdo con la conducta de alimentación, Pulido, Reyes, León, Cárdenas y Rivera (2015), mencionaron que el 50\% de los encuestados consumen productos de alto contenido graso y además no realizan actividad física significativa, en contraste con las cifras representadas en esta investigación, se observa que el $49.8 \%$ consume frituras todos los días o por lo menos de 2 a 3 veces a la semana, lo cual semeja una relación entre ambos estudios, en cuanto alimentación y actividad física.

Por otro lado, Reynaga, Fernández, Muñoz y Vera, dieron a conocer en su estudio realizado en el 2015 denominado "Percepción de comportamientos de riesgo en estudiantes universitarios del área de la salud" que 51\% de la población femenina consume cinco frutas y verduras cuatro veces/semana o más. De la población total, un $82 \%$ no fuma, $55 \%$ no consume bebidas alcohólicas y $90 \%$ no consume drogas. El $12 \%$ reporta estar estresado siempre y $72 \%$ duerme más de siete horas diarias sólo tres veces/semana o menos. Se concluye que los universitarios del área de la salud encuestados no cumplen con un estilo de vida saludable.

Lo anterior contradice la investigación presente ya que el $24.6 \%$ de la población femenina dijo consumir frutas y verduras de 4 a 6 veces por semana. Del total de los encuestados el $68.2 \%$ no fuma y no bebe alcohol y un $93.8 \%$ nunca ha consumido drogas. Además, en cuanto al estrés, el $34.9 \%$ refirió sentirse casi siempre estresado y un $45.1 \%$ duerme de 7 a 8 horas diariamente.

\section{CONCLUSIÓN}

Después del análisis de los resultados obtenidos y de acuerdo con el objetivo de este estudio se determinó que, un $48.7 \%$ los estudiantes presentan una percepción de autocuidado "bueno".

En la actualidad, el estrés es un problema que ha aumentado y que puede volverse crónico si no se interviene a tiempo, el parámetro de sentirse estresado dio como resultado que más de la mitad de los universitarios a veces se siente estresado, de los cuales el $61 \%$ menciona a veces manejar su estrés. Por otro lado, dentro de los parámetros de sueño y descanso, el $51.3 \%$ de estudiantes manifestó dormir entre 4 y 6 horas.

Aunque la autopercepción de las capacidades de autocuidado en los estudiantes tiene un nivel bueno, es necesario que durante la trayectoria académica se promuevan hábitos de 
vida saludables, ya que, en los resultados obtenidos del autocuidado en la alimentación, se demostró el $4.6 \%$ no desayuna, sin embargo, el estado nutricional es normal en la mayoría de los estudiantes.

Por ello, es importante brindar información a los jóvenes en la prevención del consumo de drogas, debido a que algunos tienen la percepción errónea de que el consumo de estas es normal y socialmente aceptable. Cabe destacar que el 93.8\% de universitarios indicó nunca haber consumido drogas ni alcohol.

Por otro lado, aunque hasta el momento no se ha dado por asentado cual es la verdadera causa de las enfermedades en el área de salud sexual, hay ciertos factores modificables que pueden ayudar a prevenirlos, como se logró identificar en el 53.8\% de los estudiantes, debido a que se realizan exámenes de salud preventivos y solo el 29.2\% lo hace cuando ya tiene alguna enfermedad.

En la actualidad se estima la promoción de la salud como la nueva salud pública dado que, su enfoque no se detiene en las enfermedades y en segundo lugar, a que proporciona herramientas para hacer frente a el cambio del perfil sociodemográfico y epidemiológico de una nación, al mismo tiempo que permitirá promover estilos de vida y espacios sociales sanos y prevenir tanto las enfermedades infecciosas como las no transmisibles. Por lo anterior, se recomienda: elaborar diferentes marcos de abordaje para los estudios cuantitativos: exploratorios, de identificación y cualitativos: teoría fundamentada, etnográficos, narrativos e investigación-acción, a fin de contar con una gama de información que muestre el tipo de autocuidado del personal de enfermería en formación en la zona Orizaba y Córdoba Veracruz.

También se debe vigilar el comportamiento de los estudiantes durante el tránsito universitario y ofrecer o fortalecer sus factores protectores, que permitan establecer redes de apoyo como las tutorías, atención integral del estudiante, programa centinela y apoyo psicopedagógico que brinde herramientas para enfrentar el fenómeno de las drogas, teniendo en cuenta que el consumo de alcohol como droga lícita y portal permite incursionar en otras de mayor riesgo.

\section{LISTA DE REFERENCIAS}

Bustos, V., Fernández, J., \& Astudillo, C, I. (2017). Autopercepción de la salud, presencia de comorbilidades y depresión en adultos mayores mexicanos: propuesta y validación de un marco conceptual simple. Biomédica, 37(1). 92-103. Recuperado 
de https://revistabiomedica.org/index.php/biomedica/article/view/3070/3458

Celis, A., \& Labrada, V. (2014). Bioestadística (3 ${ }^{a} E d$.). México: El Manual Moderno.

Diario Oficial de la Federación. [DOF]. (2013). Norma Oficial Mexicana NOM-012SSA3-2012, Que establece los criterios para la ejecución de proyectos de investigación para la salud en seres humanos. Recuperado de https://www.dof.gob.mx/nota_detalle.php?codigo=5284148\&fecha=04/01/2013

Gobierno de México. (2019). Comisión Nacional de Bioética. Comisión Nacional de Bioética. $\quad$ Recuperado de https://www.gob.mx/salud\%7Cconbioetica/es/articulos/normatividadinternacional?idiom $=\mathrm{es}$

Hernández, F., Alvarado, E., \& Pineda, E. (2013). Metodología de la investigación; Manual para el desarrollo de personal de salud. México: Limusa.

Hernández, M. L., Madueña, J., Wong, A., \& Rubio E. (2018). Estudiantes con sobrepeso y obesidad; conductas alimentarias de riesgo. Convención internacional de Salud, $\begin{array}{llll}\text { Cuba Salud, (2018). } & \text { Recuperado de }\end{array}$ http://www.convencionsalud2018.sld.cu/index.php/connvencionsalud/2018/pape r/download/438/1128

Hernández, R., Fernández, C., \& Baptista, P. (2014). Metodología de la investigación ( $6^{a}$ $E d$.$) . México: Interamericana editores.$

Martínez, D., \& Sáez, P. (2006). Conductas de Autocuidado en estudiantes universitarios residentes del complejo de hogares Huachocopihue de la UACh, 2006. (tesis de licenciatura, Universidad Austral de Chile).1-60. Recuperado de http://cybertesis.uach.cl/tesis/uach/2007/fmm188c/doc/fmm188c.pdf

Naranjo, Y., Concepción, J., \& Rodríguez, M. (diciembre, 2017). La teoría Déficit de autocuidado: Dorothea Elizabeth Orem. Gaceta Médica Espirituana, 19(3). Recuperado de http://scielo.sld.cu/pdf/gme/v19n3/GME09317.pdf

Nyombi, K., Kizito, S., Mukunya, D., Nabukalu,A., Bukama, M., Lunyera, J., Asiimwe, M., Kimuli, I., \& Kalyesubul, R. (febrero, 2016). High prevalence of hypertension and cardiovascular disease risk factors among medical students at Makerere University College of Health Sciences, Kampala, Uganda. BMC Res Notes, 9(110). 
https://www.ncbi.nlm.nih.gov/pmc/articles/PMC4756514/pdf/13104_2016_Arti cle 1924.pdf

Organización Mundial de la Salud. [OMS]. (2018). Enfermedades no transmisibles. Recuperado de https://www.who.int/es/news-room/factsheets/detail/noncommunicable-diseases

Pulido, C., Reyes, N., León, A., Cárdenas, J., Rivera, S., \& Rodríguez, A. Y. (junio, 2015). Factores de riesgo de enfermedades crónicas no transmisibles en estudiantes de ciencias básicas de la escuela medicina de la Universidad Pedagógica y Tecnológica de Colombia. Salud.hist.sanid.on-line, 10(1). Recuperado de https://www.researchgate.net/publication/298820526_FACTORES_DE_RIESG O_DE_ENFERMEDADES_CRONICAS_NO_TRANSMISIBLES_EN_ESTU DIANTES_DE_CIENCIAS_BASICAS_DE_LA_ESCUELA_MEDICINA_DE LA_UNIVERSIDAD_PEDAGOGICA

Reynaga, M., Fernández, M., Muñoz, I., \& Vera, L. (2015). Percepción de comportamientos de riesgo en estudiantes universitarios del área de la salud. Acta universitaria, 25(1). Recuperado de https://www.redalyc.org/articulo.oa?id=41641037008

Rivas, G., Feliciano, A., Verde, E., Aguilera, M., Cruz, L., Correa, E., \& Valencia, A. (2019). Autopercepción de capacidades de autocuidado para prevención de enfermedades crónicas no transmisibles en estudiantes universitarios. Enferm. Inst. Mex. Seguro Soc, 27(2). Recuperado de https://pesquisa.bvsalud.org/portal/resource/es/biblio-1015111

Sancho, A. (2016). Conocimiento y prácticas del autocuidado en salud de los estudiantes de primer ingreso, relacionado con la prevención de enfermedades no transmisibles, universidad estatal a distancia, febrero-marzo 2016. Recuperado de http://13.65.82.242:8080/xmlui/handle/cenit/1952

Secretaría de Salud. [SS]. (2019). Boletín epidemiológico Sistema Nacional de Vigilancia Epidemiologia Sistema único de Información. Recuperado de https://www.gob.mx/cms/uploads/attachment/file/455293/sem15.pdf 
Simon, L. N., López, M. G., Sandoval, R. I., \& Magaña, B. (2016). Nivel de autocuidado en estudiantes universitarios de cinco licenciaturas del área de la salud. CuiArte, 5(10), 32-43. DOI http://revistas.unam.mx/index.php/cuidarte/article/view/69114 SPSS, Inc. (2006). Guía breve de SPSS 15.0. Recuperado de https://silo.tips/queue/guiabreve-de-spss-150?\&queue id=$\underline{1 \& v=1632506921 \& u=M j g w N j o x M G E 2 O j U 6 Z j g 2 Z T o 2 Y z g 0 O m J i M D c 6 M W I y ~}$ MzphYjY3

Tamayo, M. (2014). El proceso de la investigación científica. México: LIMUSA.

Villareal E. (2015). El protocolo de investigación en las ciencias de la salud. ( $1^{a}$ Ed.). México: Trillas. 